\title{
A new, Gaia-based, catalogue of blue straggler stars in open clusters ${ }^{\star}$
}

\author{
M. J. Rain ${ }^{1}$, J. A. Ahumada ${ }^{2}$, and G. Carraro ${ }^{1}$ \\ ${ }^{1}$ Dipartimento di Fisica e Astronomia, Universita' di Padova, Vicolo Osservatorio 3, 35122 Padova, Italy \\ e-mail: mariajoserain@gmail.com \\ 2 Observatorio Astronómico, Universidad Nacional de Córdoba, Laprida 854, X5000BGR Córdoba, Argentina \\ Received 6 December 2020 / Accepted 22 February 2021
}

\begin{abstract}
Context. Blue straggler stars are exotic objects present in all stellar environments whose nature and formation channels are still partially unclear. They seem to be particularly abundant in open clusters (OCs), thus offering a unique chance to tackle these problems statistically.

Aims. We aim to build up a new and homogeneous catalogue of blue straggler stars (BSS) in Galactic OCs using Gaia to provide a more solid assessment of the membership of these stars. We also aim to explore possible relationships of the straggler abundance with the parent cluster's structural and dynamical parameters. As a by-product, we also search for possible yellow straggler stars (YSS), which are believed to be stragglers in a more advanced evolution stage.

Methods. We employed photometry, proper motions, and parallaxes extracted from Gaia DR2 for 408 Galactic star clusters and searched for stragglers within them after performing a careful membership analysis.

Results. The number of BBS emerging from our more stringent, selection criteria turns out to be significantly smaller than in previous versions of this catalogue. OCs are therefore not a preferable environment for these kinds of stars anymore. In addition, we found that BSS start to appear in clusters with ages larger than $\log (t) \sim 8.7$ and are therefore absent in very young star clusters.

Conclusions. The present catalogue supersedes the previous ones in several ways: membership assessment, number of stragglers found, and so forth. The new list includes 897 BSS and 77 YSS candidates in 408 OCs. We expect this catalogue to be the basis for a new round of studies of BSS and YSS.
\end{abstract}

Key words. catalogs - blue stragglers

\section{Introduction}

Blue straggler stars (BSS) were initially identified by Sandage (1953) in the colour-magnitude diagram (CMD) of the globular cluster M3, appearing as an extension of the cluster main sequence, blueward and above the turnoff (TO). Their presence poses a challenge for standard single-star evolution theory since stars with masses higher than that of the cluster TO should have evolved into the white dwarf regime long ago. At present, these exotic stars have been identified in essentially all stellar systems: globular clusters (GCs, Piotto et al. 2004; Salinas et al. 2012), dwarf galaxies (Momany et al. 2007), open clusters (OCs, Milone \& Latham 1994; Ahumada \& Lapasset 1995, 2007), and the field population of the Milky Way (Preston \& Sneden 2000 Santucci et al. 2015). BSS are identified according to their position in a CMD: Stars that follow the extension of the main sequence brighter than the TO are considered to be blue stragglers. In terms of magnitude, these stars typically spread from the TO to about two magnitudes brighter; in some clusters, however, the brightest BSS can be as much as 3 magnitudes above the TO. The low-luminosity limit is not always clear. For example, in OCs, stars are bluer and fainter than the TO on the zeroage main sequence (ZAMS), so they appear younger and, therefore, are BSS - for example, this includes stars 1366 and 8104

$\star$ Full Tables 3 and 4 are only available at the CDS via anonymous ftp to cdsarc.u-strasbg. fr (130.79.128.5) or via http://cdsarc. u-strasbg.fr/viz-bin/cat/J/A+A/650/A67 in NGC 188 (Geller et al. 2008) or the ones recently identified in several OCs by Leiner \& Geller (2021) using Gaia DR2. Additionally, BSS cover a broader range in colour than normal main sequence stars. Sometimes, stars located between the main sequence and the giant branch (Hertzprung gap) are also identified as BSS, or rather evolved blue stragglers 'yellow straggler stars' (YSS). Reviews on the topic can be found in Stryker (1993) and Boffin et al. (2015). By definition, BSS are expected to be more massive than the mean stellar mass of the host cluster. Their masses are usually derived from evolutionary tracks for main sequence stars, which do not include modifications to the standard theory of stellar evolution. Particularly in OCs, the photometric masses of some BSS are very different from those derived directly from their binary orbits (e.g., Sandquist et al. 2003). Today it is widely accepted that a straggler started as a normal, main sequence star that has been 'rejuvenated' by acquiring extra mass. The new, more massive star may reach an accordingly higher luminosity that can place it above the CMD TO. This increase in mass may be produced via two, non-exclusive mechanisms: mass-transfer in a close binary (McCrea 1964) or dynamically induced stellar collisions and mergers (Hills \& Day 1976; Davies et al. 1994). In the case of GCs, it has been suggested that each formation channel is favoured in different regions of the host cluster - for example, collision-induced BSS mergers would be mainly active in the dense cores (Verbunt \& Hut 1987) - and that both might be at work simultaneously (Ferraro et al. 1993; Zaggia et al. 1997; 
Mapelli et al. 2006). These scenarios, however, have not always been successful in explaining the observations. For example, BSS as mergers of previously normal main sequence stars have proposed where the progenitor of the straggler is formed in a hierarchical triple system as a result of mechanisms such as the Kozai-Lidov (Ford et al. 2000; Perets \& Fabrycky 2009; Naoz \& Fabrycky 2014) or the angular momentum loss induced by magnetic stellar winds in a close binary. This last mechanism would be responsible for at least one-third of the BSS in OCs older than 1 Gyr (Andronov et al. 2006). In old, populated and still not very dense, dynamically evolved OCs, one would expect binary evolution to be the predominant mechanism (Mathieu \& Geller 2015).

Significant advances have been made concerning binarity among BSS in OCs, based on long-term spectroscopic monitoring by Mathieu \& Geller (2015). They found a binary fraction of $70 \%$ in the blue straggler population, compared to about $25 \%$ in the normal main sequence stars; this presence of binaries shows that internal dynamical processes have not induced global stellar mergers or collisions in old OCs. Furthermore, Gosnell et al. (2014, 2015) detected and characterised some white dwarf companions to BSS in NGC 188, providing direct evidence for mass transfer events. We have less information regarding the binary properties of BSS in GCs.

In stellar clusters, BSS appear in general more centrally concentrated than the normal stellar populations. Given that they are closely related to binary evolution, it is expected that BSS should suffer mass segregation similar to the cluster's binary population and move to the centre of the cluster potential. In the 1990s, early studies gave the first hints for BSS showing this effect in GCs and some OCs (Auriere et al. 1990; Mathieu \& Latham 1986). Later studies revealed the existence of a bimodal radial distribution in these systems with a peak of stragglers at the centre, followed by a minimum at a radius $r_{\min }$, and a rise again in the outskirts of the cluster: for the models of GCs, this was done by Mapelli et al. (2006) and Milone et al. (2012); in individual GCs, this was carried out by Lanzoni et al. (2007) for M55 and Sabbi et al. (2004) for NGC 6752; and for individual OCs, this was performed by Geller et al. (2008) for NGC 188 , Carraro et al. (2014) for Melotte 66, Bhattacharya et al. (2019) for Berkeley 17, and Rain et al. (2020) for Collinder 261. The importance of this $r_{\min }$ lies on its correlation with the dynamical age of the cluster (GCs: Ferraro et al. 2012; OCs: Vaidya et al. 2020).

The study of BSS in OCs is a current high-interest subject since they give information about the number of binary systems and binaries' roles in cluster evolution. It enables an in-depth analysis of blue straggler characteristics such as frequency, orbital parameters, and masses, which are the most important diagnostic tools for determining their origin. Moreover, because of their sparse nature, OCs are good laboratories to study the nature and formation of BSS, especially with spectroscopy. So far, the most extensive survey of BSS candidates in OCs has been performed by Ahumada \& Lapasset (2007), hereafter AL07, which took into account the bibliography up to 2005. This catalogue was, in turn, a revision of the previous work by Ahumada \& Lapasset (1995), hereafter AL95, and it lists a total of 1887 blue straggler candidates in 427 OCs of all ages. AL07 found that: (i) stragglers are present in clusters of all ages; (ii) the BSS show a remarkable degree of central concentration, and (iii) the fraction of BSS increases with the richness and age of the cluster. Drawbacks of AL07 are the lack of homogeneity of the open cluster data available at the time it was published, and that the straggler candidates are mostly of an uncertain membership. Thus, while useful, these compilations are not reliable enough to allow the derivation of statistical properties of BSS. Today, an improvement in the selection of BSS has become possible thanks to the second data release of the Gaia mission (Gaia Collaboration 2018, hereafter Gaia DR2), which permits better discrimination of genuine BSS from field stars by using high-quality proper motion and parallax information. Using Gaia DR2, we can determine accurate membership and, consequently, raise the study of BSS in OCs to much more solid statistical grounds for the first time.

This paper presents a new catalogue of blue straggler stars in a large sample of OCs. It is based on the inspection of the CMDs of 408 clusters with a membership characterisation provided by the Gaia DR2 astrometric solution. The paper is organised as follows. In Sect. 2, we describe the data and the methodology used to identify the BSS and YSS. In Sect. 3 we give a detailed explanation of the contents of the catalogue itself, while in Sect. 4 we provide some general statistics. In Sect. 5 we summarise and give the conclusions of this work.

\section{Search for blue stragglers in open clusters}

This work is based on identifying blue straggler candidates in colour-magnitude diagrams of galactic OCs of all ages. This is a new compilation, for which we made use of the recent Gaia DR2 survey. For this catalogue, we also searched for YSS and introduced some methodological improvements. This section is devoted to explaining how the compilation was performed, while the catalogue's proper description is in Sect. 3.

\subsection{List of clusters}

From the original list of AL07, we extracted 389 clusters, to which we added 19 recently discovered ones with the Gaia mission, for a grand total of 408; they all appear in Cantat-Gaudin et al. (2018). This way, we dealt with a sample of clusters very similarly to those in the previous catalogues. We note, however, that the list of clusters can be easily expanded. Table 1 lists all clusters that are included in AL07 but not here; they are not considered to be bona fide clusters, mainly according to a non-compliance with two simple conditions using the Gaia DR2 astrometric solution: (i) their propermotion dispersions correspond to a physical velocity dispersion inferior to $5 \mathrm{~km} \mathrm{~s}^{-1}$; and (ii) their observed proper-motion dis-

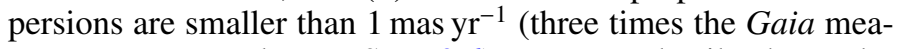
surement errors; also see Sect. 2.6). For more details, the reader is referred to Cantat-Gaudin \& Anders (2020). Furthermore, our sample does not include two well known and close clusters, Hyades (Melotte 25) and Coma (Melotte 111), given their large extension on the sky and the more sophisticated membership determination technique required to identify their BSS properly. On the other hand, the clusters listed for the first time are in Table 2.

\subsection{Milky Way open clusters with Gaia DR2 and membership criteria}

The survey Gaia DR2 provides a precise astrometric solution (RA, Dec, $\mu_{\alpha^{*}}\left(=\mu_{\alpha} \cos \delta\right), \mu_{\delta}$, and $\left.\varpi\right)$ for $\sim 1.7$ billion objects. In addition, it contains photometric data in the $G$-band for all sources, while data in the $G_{\mathrm{BP}}$ - and $G_{\mathrm{RP}}$-bands are available for $80 \%$ of the sources $(\sim 1.4$ billion objects). The limit for faint magnitudes is $G \approx 21$, while the bright limit for $G$ is around 13 . 
Table 1. Open clusters with entries in AL07, but not included in this work.

\begin{tabular}{lcc}
\hline \hline Berkeley 42 & Berkeley 64 & Berkeley 66 \\
\hline Bochum 1 & Bochum 2 & Bochum 7 \\
Bochum 10 & Bochum 14 & Collinder 96 \\
Collinder 97 & Collinder 121 & Collinder 223 \\
Collinder 228 & Coma & Dolitze 25 \\
Feinstein 1 & Hogg 16 & Hogg 22 \\
Hyades & IC 2944 & NGC 133 \\
NGC 1252 & NGC 1931 & NGC 1976 \\
NGC 2175 & NGC 2384 & NGC 2467 \\
NGC 3247 & NGC 6200 & NGC 6514 \\
NGC 6530 & NGC 6604 & Pismis 24 \\
Ruprecht 46 & Ruprecht 55 & Ruprecht 120 \\
Stock 13 & Trumpler 24 & Trumpler 27 \\
\hline
\end{tabular}

Table 2. Open clusters not included in previous versions of the catalogue.

\begin{tabular}{lcc}
\hline \hline Gulliver 1 & Gulliver 4 & Gulliver 40 \\
\hline Gulliver 12 & Gulliver 51 & Gulliver 52 \\
Gulliver 21 & Gulliver 55 & Coin 2 \\
Gulliver 23 & Coin 15 & Coin 35 \\
Gulliver 27 & Trumpler 11 & Trumpler 20 \\
Gulliver 36 & Waterloo 7 & Pozzo 1 \\
Gulliver 39 & & \\
\hline
\end{tabular}

The calibration uncertainties reached on the individual observations are 2,5 , and $3 \mathrm{mmag}$ for $G, G_{\mathrm{BP}}$, and $G_{\mathrm{RP}}$, respectively. The sources present a median uncertainty in parallax and position of about 0.04 mas for bright sources ( $G<14$ mag), 0.1 mas at $G=17 \mathrm{mag}$, and $0.7 \mathrm{mas}$ at $G=20 \mathrm{mag}$. In the proper motion components, the corresponding uncertainties are $0.05,0.2$, and

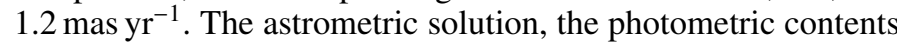
and validation, and the properties and validation of radial velocities are described in Lindegren et al. (2018), Evans et al. (2018), and Katz et al. (2019). The homogeneity and quality of Gaia data allow us to reach an unprecedented level of detail in CMDs, particularly in the context of OCs, where accurate parallax information has often been lacking.

We took advantage of the selection of cluster members carried out by Cantat-Gaudin et al. (2018), hereafter CG18, and updated by Cantat-Gaudin \& Anders (2020), hereafter CG20. The last version contains 1481 clusters and provides coordinates, proper motions, parallaxes, and distances for all of them. To assign membership probabilities $\left(P_{\text {memb }}\right)$, CG18 and CG20 applied the membership assignment code UPMASK $^{1}$ to the Gaia DR2 data in the field of each cluster. This field has a radius twice as large as the value $r_{\text {DAML02 }}$ reported by Dias et al.

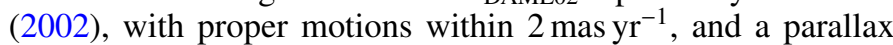
within 0.3 mas, of those of the cluster centroid $\left(\mu_{\alpha^{*}}, \mu_{\delta}, \varpi\right)$. Since

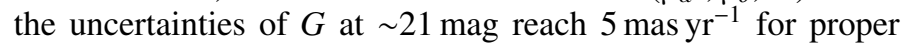
motions, and 2 mas for parallaxes, the Cantat-Gaudin catalogue includes only stars with $G \leq 18$, which corresponds to typical

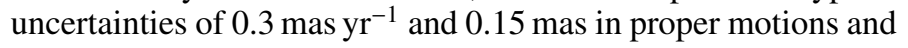
parallax. See our discussion in Sect. 2.6.

\footnotetext{
1 Unsupervised Photometric Membership Assignment in Stellar Clusters.
}

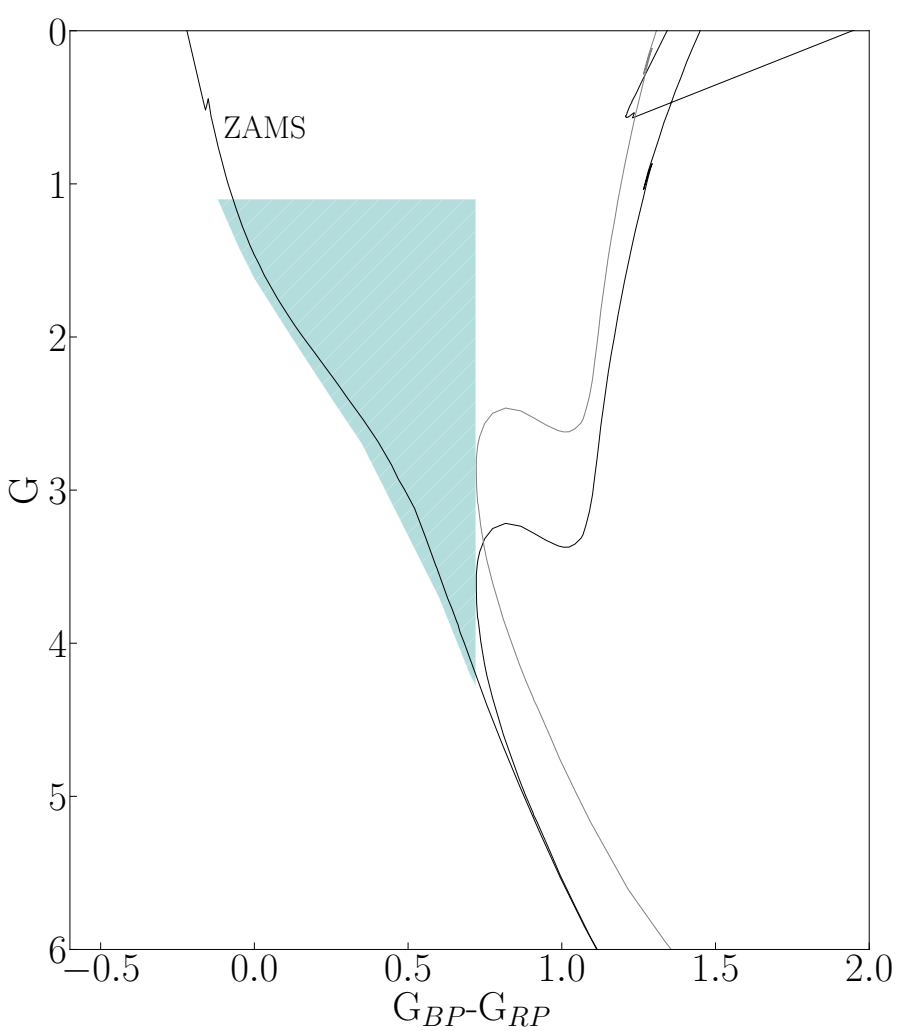

Fig. 1. Schematic colour-magnitude diagram for an old open cluster; the isochrone corresponds to $\log ($ age $)=9.8$. The blue straggler area is shaded in blue. The grey line indicates the equal-mass binary loci.

\subsection{Identification of the blue stragglers}

Formally, a blue straggler was defined from its position in a $\mathrm{CMD}$, that is, it is bluer and brighter than the TO, on or near the parent cluster ZAMS. Following AL95, AL07, and R20, we assumed a cluster star to be a blue straggler candidate if it appears on a specific area of the CMD (see Fig. 1). This region is bounded on the blue side by the ZAMS and the red side by the TO colour and the binary sequence. The lower limit corresponds to the magnitude at which the observed sequence of the cluster detaches from the ZAMS. In principle, we did not adopt a bright limit (but see Sect. 2.4).

For more details, we refer the reader to the analogous definitions in AL95 and AL07, which are the models for the present one. To identify the BSS candidates, the procedure for each cluster was the following; we note that the reader may also find Sect. 2.2.1 of AL07 useful:

1. The photometric data for all CG18 members (Sect. 2.2) with probabilities of $P_{\text {memb }} \geq 50 \%$ were plotted in a $G$ versus $\left(G_{\mathrm{BP}}-G_{\mathrm{RP}}\right)$ diagram.

2. An approximate matching of a PARSEC theoretical isochrone (Bressan et al. 2012), with the Gaia DR2 passbands of Evans et al. (2018), was then performed on the main sequence and TO, and eventually on the red giant branch (RGB) and red clump if present. Cluster parameters, such as metallicity, extinction, and the age of DAML02, were first chosen. When the cluster was not listed in DAML02 or when the matching of the isochrone was unsatisfactory, parameters from Bossini et al. (2019) and Monteiro \& Dias (2019) were used instead. However, the distance was always taken from CG18. Sometimes a refinement of the parameters was necessary; in such cases, we kept the distance and extinction 


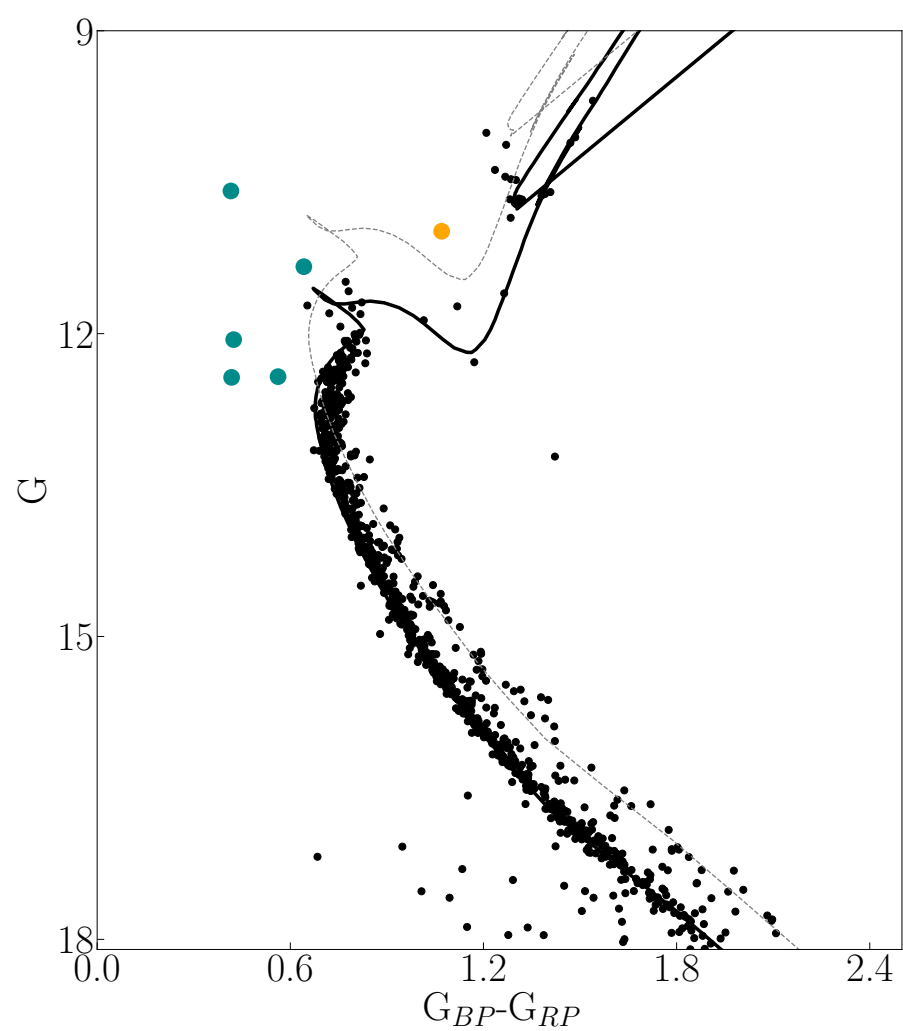

Fig. 2. CMD of the open cluster IC 4651, built from Gaia DR2 photometry. Black filled circles are all the stars from CG18 with $P_{\text {memb }} \geq 50 \%$. Blue filled circles and yellow filled circles are the catalogued blue and YSS, respectively. The black solid line represents the PARSEC isochrone of solar metallicity and $\log ($ age $)=9.27$ (Bressan et al. 2012), set at $E(B-V)=0.36$ and $(m-M)_{0}=9.82$. The second curve is the same isochrone displaced $0.75 \mathrm{mag}$, which helps constrain the region of the binaries. See also Table 4.

values fixed and varied the age - that is to say, we changed the isochrone, adopting the solar value when no metallicity was listed in the literature. Based on these parameters' variation, we picked the appropriate PARSEC isochrone by eye, from a grid spaced 0.05 in $\log ($ age $)$. These changes are reported in the catalogue Notes (see Sect. 3.4).

3. The equal-mass binary loci - obtained by displacing the isochrone by $0.75 \mathrm{mag}$ upward - and the ZAMS were also plotted. The binary loci help constrain the region expected to be populated by binaries made of normal main sequence TO stars.

4. Finally, the stragglers' candidates were singled out in the corresponding region of the CMD. As an example, Fig. 2 shows this selection in the CMD of IC 4651.

The aforementioned procedure was executed, taking the following considerations into account. First, stars located slightly towards the blue of the ZAMS were also regarded as straggler candidates. Second, not all the stars fainter than the cluster TO and located between the ZAMS and the isochrone were included: Only objects significantly detached from the cluster isochrone, for instance $\sim 0.03 \mathrm{mag}$ as a minimum and down to $0.5-1.5 \mathrm{mag}$ at most below the TO, were listed. This magnitude cut is consistent with our limit of $G \approx 18$ for the oldest and distant clusters in our sample (see Sect. 2.6 and CG18). Third, although we took the TO colour for the straggler region's red limit, an arbitrary cut-off in colour was imposed for some clusters similarly as in AL07. Redder stars were considered as possible
YSS (YSS, Sect. 2.5). Finally, in this work, as in AL07, stars on the 'blue hook' were not regarded as stragglers, given the dependence of this feature's extension and shape with the stellar models adopted for the isochrone. In this sense, the adoption of the equal-mass binary sequence (see, e.g., Fig. 2) was beneficial. This sequence wraps and helps to constrain the blue hook region of the cluster, when possible, since here normal photometric binaries may fall, increasing the straggler population spuriously.

\subsection{Massive stragglers}

Although we decided not to define a bright limit in the BS area (Sect. 2.3 and Fig. 1), there is actually a sort of frontier given by the theory of mass transfer in close binaries of $2.5 \mathrm{mag}$ above the TO (Chen \& Han 2009; McCrea 1964): This is the luminosity that would reach the complete merger of two identical (same mass) main sequence stars at the TO. However, there is evidence of brighter, bona fide BSS similar to, for instance, star 677 in NGC 7789 (Breger \& Wheeler 1980). Stars similar to this could be the result, for example, of a three-body interaction and subsequent merger. We flagged these stars as 'massive' to identify them as subjects of further, specific research. To locate them, we used isochrones whose TOs are $2.5 \mathrm{mag}$ brighter than those of the isochrones used to match the CMDs. In practice, this implied adopting an upper limit of about 1-1.5 mag above the $2.5 \mathrm{mag}$ frontier.

\subsection{Yellow stragglers}

We name YSS the objects with colours between those of the TO and the RGB, and they are brighter than the subgiant branch (Clark et al. 2004) ${ }^{2}$. They have been photometrically and spectroscopically identified in both open and globular clusters (Sales Silva et al. 2014; da Silveira et al. 2018; Rain et al. 2021; Martinez et al. 2020), and they are usually explained as evolved BSS - post-main sequence stars more massive than the TO on their way to the RGB (Mathieu et al. 1990 and references therein). For example, four YSS have been identified in M67, one of them with a Helium white dwarf (WD) companion, indicating that it is an evolved straggler that formed from mass transfer, having an RGB star as a donor (Landsman \& Stecher 1997; Landsman \& Simon 1998). Leiner et al. (2016) reported the first asteroseismic mass and radius measurements of the yellow straggler S1237 in M67; they argue that it might be the result of a stellar collision or a binary merger. The methodology followed for finding YSS was similar to the one used for BSS (Sect. 2.3). Figure 3 shows the region where these stars were searched for. This region is limited to the left by the TO colour or red limit depending on the cluster. Below and to the right by the grey equal-mass photometric binary sequence, which corresponds to the matched isochrone that moved 0.75 to brighter magnitudes and that represents the maximum brightness expected for an equal mass binary at the cluster TO. In OCs, however, the YSS regime can also contain the binaries' product of mass transfer or mergers (models of Tian et al. 2006 and Chen \& Han 2008),

\footnotetext{
2 In the literature, there is a potential source of confusion regarding the naming of these stars since the 'red straggler' term has also been systematically used for stars lying between the blue straggler area and the RGB (e.g., Eggen 1983; Eggen \& Iben 1988; Landsman \& Stecher 1997; Landsman \& Simon 1998; Albrow et al. 2001). In this work, we chose to designate these objects as 'yellow stragglers', and we encourage the community to adopt this nomenclature.
} 


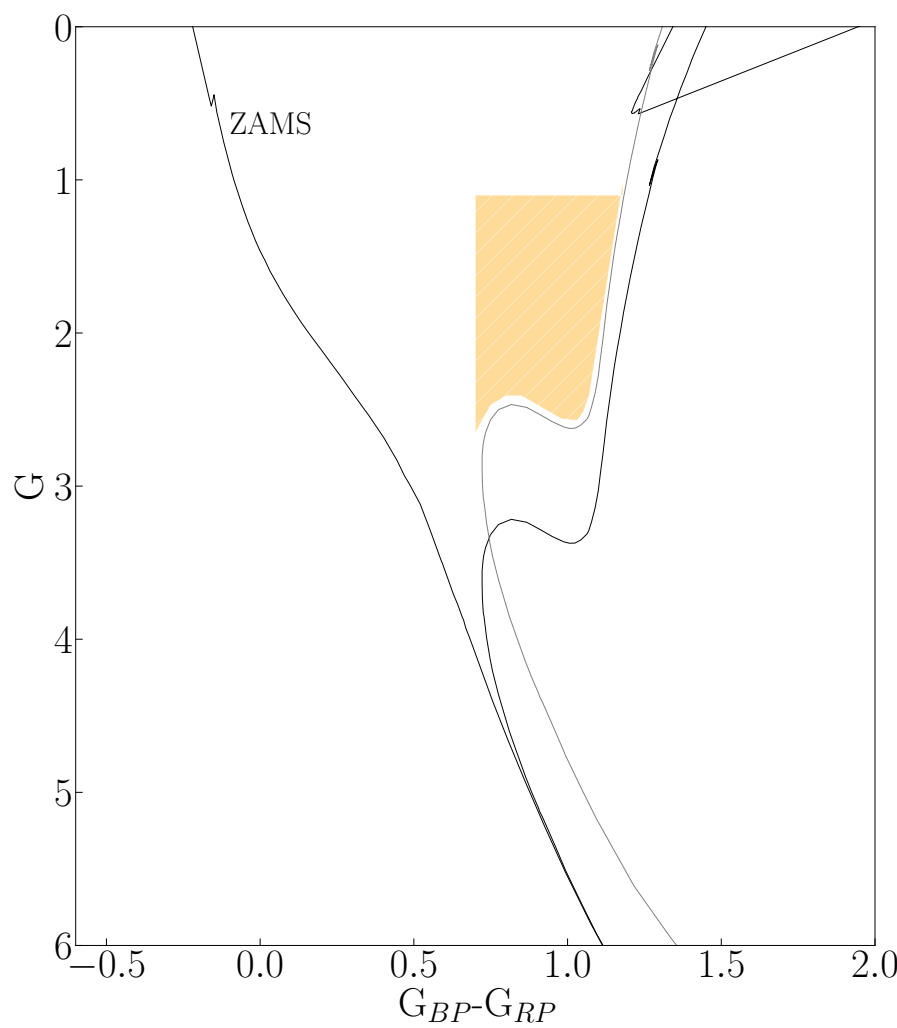

Fig. 3. Yellow straggler area in a schematic colour-magnitude diagram of an old open cluster $(\log ($ age $)=9.8)$. The equal-mass binary loci is also plotted.

and they can even be 'contaminated' by objects that formed by two-member stars (e.g., a red giant-main sequence star), whose combined lights photometrically place the system within the Hertzsprung gap (Mermilliod et al. 2007).

Finally, although no upper limit was defined, and considering that the CMDs can be affected by differential extinction - which may distort the clump structure-those stars with magnitudes fainter than the clump and clearly differentiated from the cluster sequences were also labelled as possible YSS.

\subsection{Limitations of Gaia DR2 photometry}

Gaia DR2 suffers from natural calibration problems and systematic errors, both for very bright and faint sources. Here, we briefly describe how these limitations can affect the completeness of our catalogue.

For faint sources ( $G \geq 17-18 \mathrm{mag}$ ), problems in the background calibration and contamination from nearby sources have been reported (Evans et al. 2018; Arenou et al. 2018). For this reason, our catalogue contains only sources brighter than $G \approx$ 18, which is the limit adopted by Cantat-Gaudin et al. (2018). This cut in terms of distance corresponds to the de-reddened magnitude of a 3-Gyr star cluster's TO at $10 \mathrm{kpc}$. Hence, we did not include a few distant and old OCs with TOs fainter than $G \approx 18$ in the catalogue because of the virtual impossibility of matching an isochrone to a CMD without a clear TO. An example is Berkeley 66, whose TO is at $G \sim 19$.

In Gaia, the sources described as 'bronze' are photometrically non-calibrated objects, without colour measurements, and for which only the $G$ magnitude is provided. They tend to prevail in high-density stellar environments. These sources cannot be represented in a CMD, and therefore they will not be cata- logued. An example is star 266 of NGC 2354, which is a $\beta$ Lyrae eclipsing binary (V* QU CMa, Lapasset \& Ahumada 1996).

On the other hand, the bright end $(G \leq 13)$ is limited by photometric calibration errors. In clusters with a TO brighter than $G \approx 12$, where saturation and flux loss becomes important, and the instrument calibration is still very provisional and problematic, some BSS may be missing. For example, IC 2602 $\left(G_{\mathrm{TO}} \sim 4\right)$ harbours the bright straggler $\theta$ Carinae (HD 93030, Walborn 1979; Nazé \& Rauw 2008), which does not appear in Gaia and therefore has not been included in our list.

\subsection{Field contamination in the straggler region}

Usually, field stars located between us and a cluster contaminate its CMDs. This is particularly true for OCs since most of them are located low onto the disc, and many are projected towards the Galaxy bulge. In fact, Carraro et al. (2008) showed that stars belonging to the young stellar population of the Milky Way disc tend to occupy the same region on the CMD as the BSS, artificially enhancing the clusters' BSS population. Cluster members, selected as in CG18 on an astrometric basis only, are expected to include a fraction of interlopers. Disc star contamination is then one of the main reasons we decided to retain only potential members with $P_{\text {memb }} \geq 50 \%$. This criterion should enhance the probability of picking up the most likely members (Carrera et al. 2019; Banks et al. 2020; Yontan et al. 2019) and providing more robust BSS statistics.

\subsection{Differential reddening}

It is well known that the patchy dust distribution in the field of view towards star clusters causes differential extinction (see, e.g., Platais et al. 2008); this manifests as a broadening of the stellar sequences in CMDs. For old OCs (age $\geq 1 \mathrm{Gyr}$ ), the effects of differential reddening are most noticeable in the TO and RGB morphologies. In particular, the Gaia photometric bands are broad enough to introduce large colour differences caused by extinction as a function of the stellar SED. These spreads in colour can introduce some dispersion in the CMD positions, affecting the selection, especially near the TO. As shown by Leiner \& Geller (2021), for clusters with low reddening values $\left(E\left(G_{\mathrm{BP}}-G_{\mathrm{RP}}\right)<0.3\right)$, it is sufficient to adopt reddenings from the literature and convert them to the Gaia passbands. On the other hand, for clusters with high reddenings, individual reddening corrections are recommended. Although a small number of clusters in our sample show high differential extinction across their field, we did not attempt to correct the photometry of the individual sources from interstellar extinction; in these cases, a warning was included in the Notes (Sect. 3.4). In Rain et al. (2021), we explored this effect in two OCs (Trumpler 5 and Trumpler 20). We conclude that their straggler populations did not change considerably despite their low-latitude Galactic position and high extinction values. We refer the reader to that study for more information regarding the methodology used to quantify this effect in OCs using Gaia DR2 data.

\section{The catalogue}

This new catalogue of BSS in OCs contains the following files: one first table with open cluster data, a second table listing blue straggler data, a third table with the yellow straggler compilation, and, finally, a file with notes and comments. 
Table 3. Excerpt of the catalogue compiling general information of the open clusters.

\begin{tabular}{|c|c|c|c|c|c|c|c|c|c|c|c|}
\hline Cluster & $\begin{array}{c}\text { RA } \\
\text { [degrees] }\end{array}$ & $\begin{array}{c}\text { Dec } \\
\text { [degrees] }\end{array}$ & $\log ($ age $)$ & $E(B-V)$ & $\begin{array}{c}\left\langle\mu_{\alpha^{*}}\right\rangle \\
{\left[\mathrm{mas} \mathrm{yr}^{-1}\right]}\end{array}$ & $\begin{array}{c}\left\langle\mu_{\delta}\right\rangle \\
{\left[\operatorname{mas}_{\mathrm{yr}}{ }^{-1}\right]}\end{array}$ & $\begin{array}{c}\langle\varpi\rangle \\
{[\mathrm{mas}]}\end{array}$ & $\begin{array}{c}\text { Distance } \\
{[\mathrm{pc}]}\end{array}$ & $N_{\mathrm{BSS}}$ & $N_{\mathrm{YSS}}$ & Notes \\
\hline NGC_7788 & 359.179 & 61.395 & 7.26 & 0.52 & -3.112 & -1.762 & 0.300 & 3039.2 & 0 & 0 & $*$ \\
\hline King_12 & 358.265 & 61.953 & 7.85 & 0.51 & -3.416 & -1.420 & 0.296 & 3080.6 & 0 & 0 & \\
\hline King_11 & 356.912 & 68.636 & 9.04 & 1.27 & -3.358 & -0.643 & 0.262 & 3433.2 & 18 & 0 & $*$ \\
\hline King_20 & 353.305 & 58.469 & 8.30 & 0.65 & -2.686 & -2.585 & 0.496 & 1903.8 & 0 & 0 & \\
\hline NGC_7654 & 351.195 & 61.590 & 8.20 & 0.57 & -1.938 & -1.131 & 0.596 & 1600.1 & 0 & 0 & \\
\hline Berkeley_99 & 350.260 & 71.778 & 9.50 & 0.30 & -3.139 & -0.359 & 0.137 & 6029.4 & 5 & 1 & $*$ \\
\hline Mrk_50 & 348.806 & 60.448 & 7.09 & 0.81 & -3.465 & -2.560 & 0.323 & 2837.6 & 0 & 0 & \\
\hline NGC_7510 & 347.767 & 60.579 & 7.35 & 0.90 & -3.664 & -2.193 & 0.286 & 3177.5 & 0 & 0 & \\
\hline King_19 & 347.053 & 60.523 & 8.55 & 0.54 & -4.838 & -2.651 & 0.343 & 2687.7 & 1 & 0 & \\
\hline King_10 & 343.748 & 59.170 & 7.44 & 1.13 & -2.722 & -2.088 & 0.259 & 3478.1 & 0 & 0 & \\
\hline NGC_7419 & 343.579 & 60.814 & 7.15 & 2.02 & -2.759 & -1.601 & 0.280 & 3235.9 & 0 & 0 & \\
\hline NGC_7380 & 341.817 & 58.125 & 7.07 & 0.60 & -2.517 & -2.144 & 0.333 & 2765.6 & 0 & 0 & \\
\hline Berkeley_96 & 337.478 & 55.408 & 7.60 & 0.17 & -3.503 & -2.994 & 0.252 & 3560.6 & 0 & 0 & \\
\hline NGC_7261 & 335.056 & 58.128 & 8.20 & 0.88 & -3.932 & -2.909 & 0.278 & 3261.9 & 0 & 0 & \\
\hline NGC_7243 & 333.788 & 49.830 & 8.00 & 0.18 & +0.433 & -2.857 & 1.116 & 873.3 & 0 & 0 & \\
\hline NGC_7235 & 333.083 & 57.271 & 7.00 & 0.81 & -2.381 & +2.935 & 0.152 & 5536.9 & 0 & 0 & $*$ \\
\hline NGC_7209 & 331.224 & 46.508 & 8.53 & 0.17 & +2.255 & +0.283 & 0.820 & 1177.7 & 0 & 0 & $*$ \\
\hline NGC_7160 & 328.448 & 62.589 & 7.27 & 0.37 & -3.472 & -1.378 & 1.050 & 926.7 & 0 & 0 & \\
\hline IC_5146 & 328.372 & 47.246 & 6.00 & 0.59 & -2.910 & -2.490 & 1.213 & 805.0 & 0 & 0 & \\
\hline NGC_7142 & 326.290 & 65.782 & 9.55 & 0.35 & -2.747 & -1.288 & 0.392 & 2376.4 & 10 & 1 & $*$ \\
\hline$\vdots$ & $\vdots$ & $\vdots$ & $\vdots$ & $\vdots$ & $\vdots$ & $\vdots$ & $\vdots$ & $\vdots$ & $\vdots$ & $\vdots$ & $\vdots$ \\
\hline
\end{tabular}

Notes. The full version of this table is available at the CDS. In the table, the clusters are sorted by right ascension.

Table 4. Blue straggler candidates in the open cluster IC 4651 (see also Fig. 2).

\begin{tabular}{|c|c|c|c|c|c|c|c|c|c|c|}
\hline Cluster & Gaia DR2 Source Id. & $\begin{array}{c}\text { RA } \\
\text { [degrees] }\end{array}$ & $\begin{array}{c}\text { Dec } \\
\text { [degrees] }\end{array}$ & $\begin{array}{c}\mu_{\alpha^{*}} \\
{\left[\mathrm{mas} \mathrm{yr}^{-1}\right]}\end{array}$ & $\begin{array}{c}\mu_{\delta} \\
{\left[\mathrm{mas} \mathrm{yr}^{-1}\right]}\end{array}$ & $\begin{array}{c}\varpi \\
{[\mathrm{mas}]}\end{array}$ & $G$ & $G_{\mathrm{BP}}-G_{\mathrm{RP}}$ & $P_{\text {memb }}$ & $\begin{array}{c}r \\
{[\operatorname{arcmin}]}\end{array}$ \\
\hline IC_4651 & 5949561772753237248 & 260.920 & -49.9 & -2.29 & -5.386 & 1.2379 & 11.23 & 0.64 & 0.6 & 11.61 \\
\hline IC_4651 & 5949565616715032064 & 261.128 & -49.9 & -3.36 & -5.255 & 1.0841 & 11. & 0.42 & 0.6 & 3.21 \\
\hline IC_4651 & 5949553522087135616 & 261.231 & -49.9 & -2.10 & -4.683 & 1.0457 & 12.33 & 0.41 & 0.7 & 2.86 \\
\hline IC_4651 & 5949553835654195968 & 261.273 & -49.9 & -2.26 & -4.762 & 1.1896 & 10.48 & 0.41 & 1.0 & 2.93 \\
\hline IC_4651 & 5949584170973723520 & 261.507 & -49.7 & -2.02 & -5.612 & 1.1522 & 12.32 & 0.56 & 0.6 & 17.13 \\
\hline
\end{tabular}

Notes. This is an extract of the full table, which is available at the CDS.

\subsection{First table: Open cluster data}

The cluster parameters were mainly taken from DAML02, CG18, and Bossini et al. (2019). For clusters recently discovered, we used the data provided by Monteiro \& Dias (2019). The parameters listed for each cluster are as follows: the equatorial coordinates (J2000.0), the logarithm of the age, the reddening, the mean proper motions, the parallaxes, the distance, the number of BSS and YSS candidates with $P_{\text {memb }} \geq 50 \%$, and eventual notes (cf. Sect. 3.4). In total, the table comprises twelve columns. Table 3 is an extract of the full Table, which is available at the CDS.

\subsection{Table 2: Blue stragglers in open clusters}

In this table, we list information on every identified straggler candidate. In total, the table contains eleven columns. The first column gives the cluster's common name, while the second column indicates the Gaia DR2 identification. Columns three to seven list the coordinates, the individual proper motions, and the parallaxes. Columns eight and nine provide the $G$ magnitude and the $\left(G_{\mathrm{BP}}-G_{\mathrm{RP}}\right)$ index. Column ten indicates the membership probability by CG18. Column eleven lists the distance from the cluster centre. As an example, Table 4 shows the entries for the cluster IC 4651; the full Table is available at the CDS.

\subsection{Table 3: Yellow stragglers in open clusters}

This table lists information on every identified yellow straggler candidate. It contains eleven columns in the same format as in Table 4 (see Sect. 3.2 for more information).

\subsection{Notes}

This file gathers information that clarifies, complements, or simply adds content to the Tables previously mentioned. 
Table 5. Open clusters harbouring YSS.

\begin{tabular}{lcclcc}
\hline \hline Cluster & $\log ($ age $)$ & $N_{\text {YSS }}$ & Cluster & $\log ($ age $)$ & $N_{\text {YSS }}$ \\
\hline NGC 2477 & 8.80 & 4 & Berkeley 69 & 8.95 & 1 \\
Collinder 261 & 9.95 & 3 & King 7 & 8.82 & 1 \\
Berkeley 20 & 9.78 & 3 & Trumpler 20 & 9.11 & 1 \\
NGC 6253 & 9.70 & 3 & Berkeley 70 & 9.67 & 1 \\
NGC 2437 & 8.40 & 3 & NGC 5823 & 8.90 & 1 \\
Berkeley 19 & 9.40 & 3 & Berkeley 29 & 9.02 & 1 \\
NGC 2141 & 9.23 & 3 & Pismis 3 & 9.02 & 1 \\
Ruprecht 75 & 9.15 & 3 & IC 1311 & 9.20 & 1 \\
King 5 & 9.10 & 3 & NGC 1245 & 9.00 & 1 \\
NGC 2158 & 9.02 & 3 & NGC 7142 & 9.55 & 1 \\
Berkeley 23 & 8.90 & 3 & Melotte 66 & 9.53 & 1 \\
Trumpler 5 & 9.60 & 2 & NGC 2243 & 9.03 & 1 \\
Berkeley 39 & 9.90 & 2 & NGC 3680 & 9.07 & 1 \\
King 2 & 9.78 & 2 & NGC 2192 & 9.15 & 1 \\
Berkeley 32 & 9.70 & 2 & NGC 6705 & 8.40 & 1 \\
NGC 1193 & 9.70 & 2 & NGC 752 & 9.16 & 1 \\
NGC 2682 & 9.45 & 2 & IC 4651 & 9.05 & 1 \\
NGC 1798 & 9.25 & 2 & NGC 2354 & 9.18 & 1 \\
IC 166 & 9.09 & 2 & Berkeley 99 & 9.50 & 1 \\
NGC 2204 & 9.03 & 2 & NGC 3114 & 8.09 & 1 \\
Tombaugh 2 & 9.01 & 2 & NGC 7654 & 8.20 & 1 \\
Berkeley 81 & 9.00 & 2 & & & \\
\hline
\end{tabular}

\section{Statistics}

\subsection{Comparison with AL95 and ALO7}

In total, 897 blue straggler candidates were identified in the 408 OCs investigated. The number of clusters with at least one blue straggler is $111(27.20 \%)$. In comparison with AL95, where 959 BS candidates were found in 390 clusters, and where the number of clusters with any stragglers was $225(57.7 \%)$, our percentage is $\sim 30 \%$ less. The respective numbers for AL07 are as follows: 1887 stragglers, 427 clusters, and 199 clusters with stragglers (46.6\%); here the difference is $\sim 20 \%$. It can be safely assumed that a good part of this results from the greatly improved membership information available for the present work because of Gaia. Regarding the YSS, 77 candidates were identified in 43 clusters $(10.53 \%)$, with most of them hosting only one yellow straggler. These clusters and their $N_{\text {YSS }}$ are listed in Table 5. No straggler candidates were found in clusters with $\log ($ age $) \leq 7.5$. In AL95 and AL07, some stragglers - albeit few - were identified in young clusters; this may reflect a bias towards bright magnitudes (see the case of IC 2602 in Sect. 2.6), besides membership considerations.

In Table 6, we list the clusters with $N_{\mathrm{BS}} \geq 10$. In apparent contrast to our remarks in the preceding paragraph, it is interesting to note that some of the OCs show a greater number of BSS candidates in our catalogue than in AL07. For example, we found that Trumpler 5 has the largest straggler population (Rain et al. 2021), but AL07 listed only 70 stars, of which just four were classified as of 'type 1' - that is to say, bona fide stragglers given the then available membership information. The discrepancy may result from the different areas searched for BSS, a radius of $8^{\prime}$ in AL07, and 30' in the present work. However, for most of the clusters, the opposite occurs, and the number of catalogued BSS decreases considerably. Most of the objects in Table 6 were also targeted by AL07 as the richest in BSS. As for the age of these clusters, all of them are old ( $\log ($ age $) \geq 9.0)$, just as AL95 and AL07 found. A comparison between our catalogue and AL07 is possible only for those sources with RA and Dec
Table 6. Open clusters with the largest absolute population of blue stragglers $\left(N_{\mathrm{BSS}} \geq 10\right)$ in comparison with AL07.

\begin{tabular}{lccc}
\hline \hline Cluster & log(age) & $N_{\text {BSS }}$ & $N_{\text {BSS }}($ AL07) \\
\hline Trumpler 5 & 9.60 & 103 & 70 \\
Collinder 261 & 9.95 & 53 & 54 \\
NGC 6791 & 9.92 & 48 & 75 \\
NGC 2158 & 9.02 & 39 & 40 \\
Berkeley 18 & 9.63 & 32 & 126 \\
Berkeley 32 & 9.70 & 27 & 37 \\
NGC 1798 & 9.25 & 27 & 24 \\
Tombaugh 2 & 9.25 & 27 & $\ldots$ \\
King 2 & 9.78 & 26 & 30 \\
NGC 188 & 9.88 & 22 & 24 \\
Berkeley 17 & 10.0 & 20 & 31 \\
Berkeley 21 & 9.34 & 20 & 51 \\
NGC 2141 & 9.23 & 18 & 24 \\
King 11 & 9.04 & 18 & 27 \\
Berkeley 39 & 9.90 & 18 & 43 \\
NGC 7789 & 9.52 & 16 & 22 \\
NGC 6819 & 9.36 & 15 & 29 \\
NGC 6253 & 9.70 & 14 & 27 \\
Melotte 66 & 9.53 & 14 & 35 \\
NGC 2243 & 9.03 & 14 & 09 \\
NGC 2506 & 9.00 & 14 & 15 \\
Berkeley 12 & 9.60 & 13 & 15 \\
Berkeley 19 & 9.40 & 13 & 01 \\
NGC 1193 & 9.70 & 12 & 19 \\
Berkeley 70 & 9.67 & 12 & 64 \\
Trumpler 20 & 9.11 & 12 & $\ldots$ \\
NGC 2682 & 9.45 & 11 & 30 \\
NGC 7142 & 9.55 & 11 & 37 \\
\hline
\end{tabular}

information available. These were obtained from $\mathrm{WEBDA}^{3}$ or any data linked to ADS. For the sources we matched, we found large inconsistencies between our catalogue and AL07. First, for individual clusters, the percentage of BSS found to be nonmembers according to our criteria (Sect. 2.2) is about $10-60 \%$ of the AL07 BSS. However, there are cases such as NGC 2477, whose AL07 BSS are all members, but that appear concentrated around the TO and sub-giant branch in the Gaia CMD. On the other hand, for close and very well studied clusters with spectroscopically confirmed BSS, it is possible to retrieve about $70 \%$ and in some cases up to $100 \%$, as Vaidya et al. (2020) have demonstrated. We are aware that some BSS are lost given our stringent and conservative selection criteria. Although we did not attempt to estimate the number of missed BSS, we are confident that this is small because our choice of stars with $P_{\text {memb }} \geq$ $50 \%$ captures the majority of the cluster members, as found by other authors (Cantat-Gaudin et al. 2018; Carrera et al. 2019; Banks et al. 2020; Yontan et al. 2019).

\section{2. $N_{\mathrm{BSS}} / \mathrm{N}_{\mathrm{MSS}}$ versus age}

Figure 4 shows the ratio $N_{\mathrm{BSS}} / N_{\mathrm{MSS}}$ versus cluster age, in a logarithmic scale, for 107 OCs. The $N_{\text {MSS }}$ is the number of cluster main sequence stars up to 1 magnitude below the TO, adopted as a proxy of the cluster richness. The clusters were chosen first by visually inspecting every CMD, built with stars of $P_{\text {memb }} \geq$ $80 \%$, and then by retaining those that showed well-defined

3 https://webda.physics.muni.cz/ 


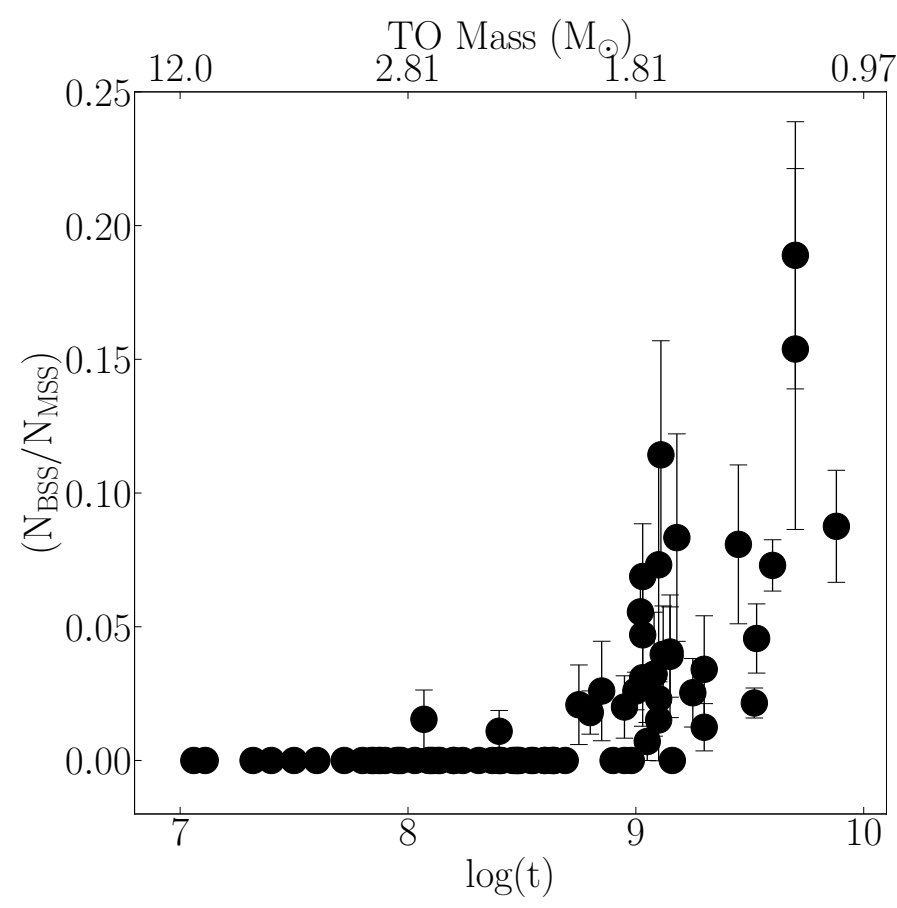

Fig. 4. $N_{\mathrm{BSS}} / N_{\mathrm{MSS}}$ as a function of the age in logarithmic scale. Only 107 clusters are included. Errors are Poisson.

evolutionary phase signatures - that is main sequence, binary sequence, and red clump when it was possible - and those that were well-matched to the corresponding isochrone. In the top $x$-axis of the figure, the mass of the cluster TO for a given age is shown. Star counts are in Table 7.

The results can be summarised as follows. The ratio is approximately constant for young OCs until $\log ($ age $) \sim 8.7$ ( $\sim 500 \mathrm{Myr})$, followed by a steep increase for older clusters. Previous works attempted to discern if this correlation between $N_{\text {BSS }} / N_{\text {MSS }}$ and the age is true and related with some intrinsic mechanism that would produce more stragglers in old clusters. Surprisingly, our results are more similar to AL95 than AL07 when the number of stragglers is normalised to main sequence stars. In AL07, the absolute number of stragglers grows with the cluster age starting from $\log ($ age $) \sim 6.5$, while in AL95, two trends are present, one is approximately constant for young clusters and the second one grows with age. One scenario to explain the difference observed between both figures is related to the lack of BSS in young OCs and the confusion of defining an accurate TO in the CMD, which means that it is hard to distinguish MS stars from BSS, misclassifying them as stragglers. Concerning the sudden increase, it was proposed by AL07 that the number of stragglers observed in old OCs is a consequence of mechanisms such as mass transfer in close binaries. This remains an open issue that requires further investigation.

\section{Conclusions}

We have presented a new catalogue of blue and yellow stragglers in OCs, under the necessity of updating the previous versions based on two important facts. First, the burst of all-sky and large surveys over the whole range of the electromagnetic spectrum provides new, homogeneous data for an enormous amount of objects, allowing their study over larger sky areas. Second, the fact that there is the need to identify straggler candidates more reliably and accurately, not merely based on their position
Table 7. Clusters with the largest relative population of blue stragglers $\left(N_{\mathrm{BSS}} / N_{\mathrm{MSS}} \geq 0.01\right)$.

\begin{tabular}{lcccc}
\hline \hline Cluster & $\log (\mathrm{age})$ & $N_{\text {BSS }}$ & $N_{\text {MSS }}$ & $N_{\text {BSS }} / N_{\text {MSS }}$ \\
\hline Berkeley 32 & 9.70 & 17 & 90 & 0.19 \\
NGC 6253 & 9.70 & 06 & 39 & 0.15 \\
Trumpler 20 & 9.11 & 08 & 70 & 0.11 \\
NGC 188 & 9.88 & 19 & 217 & 0.09 \\
NGC 2354 & 9.18 & 05 & 60 & 0.08 \\
NGC 2682 & 9.45 & 08 & 99 & 0.08 \\
King 5 & 9.10 & 03 & 41 & 0.07 \\
Trumpler 5 & 9.60 & 62 & 850 & 0.07 \\
NGC 2243 & 9.03 & 13 & 189 & 0.07 \\
NGC 2158 & 9.02 & 28 & 504 & 0.06 \\
NGC 2204 & 9.03 & 07 & 149 & 0.05 \\
Melotte 66 & 9.53 & 13 & 285 & 0.05 \\
Collinder 110 & 9.15 & 06 & 148 & 0.04 \\
Melotte 71 & 9.12 & 05 & 126 & 0.04 \\
NGC 2627 & 9.15 & 03 & 77 & 0.04 \\
NGC 2420 & 9.30 & 03 & 88 & 0.03 \\
NGC 6005 & 9.08 & 02 & 62 & 0.03 \\
NGC 2660 & 9.03 & 03 & 97 & 0.03 \\
NGC 2506 & 9.00 & 14 & 539 & 0.03 \\
NGC 6940 & 8.85 & 02 & 77 & 0.03 \\
NGC 2112 & 9.25 & 04 & 158 & 0.03 \\
NGC 7044 & 9.10 & 06 & 260 & 0.02 \\
NGC 7789 & 9.52 & 15 & 698 & 0.02 \\
NGC 6134 & 8.95 & 03 & 150 & 0.02 \\
NGC 2477 & 8.80 & 05 & 279 & 0.02 \\
NGC 6067 & 8.07 & 02 & 130 & 0.02 \\
NGC 3960 & 9.10 & 01 & 66 & 0.02 \\
NGC 6603 & 8.30 & 01 & 96 & 0.02 \\
NGC 6939 & 9.30 & 02 & 161 & 0.01 \\
NGC 6705 & 8.07 & 02 & 183 & 0.01 \\
IC 4651 & 9.04 & 01 & 141 & 0.01 \\
\hline
\end{tabular}

Notes. Only stars with $P_{\text {memb }} \geq 80 \%$ were considered.

in the CMD, but also using the more accurate astrometric membership provided by Gaia. We thus defined homogeneous criteria for the selection of the BSS to derive proper-motion-cleaned BSS catalogues in all our OCs. In this edition, 897 stragglers were identified in 111 clusters of a total of 408 OCs, of which 19 are recently discovered objects that are not present in the catalogue AL07; on the other hand, 39 clusters listed in AL07 are not present in this new version. Regarding yellow stragglers counts, 77 YSS were identified in 43 OCs. The proper motion decontamination allowed an unambiguous selection of YSS in the CMD, which had not been possible in previous BSS studies in OCs, given the high field star contamination that the Galactic disc causes in the CMDs. We want to remark that this is the very first catalogue containing this information.

We also want to draw attention to another important difference that the reader will find between this work and AL07: The classification of straggler candidates in type 1 and type 2 defined by AL07 is not included in our catalogue. We instead give the coordinates, the astrometric solution, and the distance to the cluster centre of each BSS and YSS.

We hope that this new compilation will be a useful reference for, and give a new boost to, studies of blue straggler and yellow straggler populations in OCs. We also believe it can be of great use, particularly for new follow-up observations of radial velocities that will provide a strong confirmation of our selected 
straggler candidates' membership and improve our knowledge of these interesting objects.

Acknowledgements. MJ. R. is supported by CONICY PFCHA through Programa de Becas de Doctorado en el extranjero- Becas Chile/2018-72190617 G. C. acknowledges funding from Italian Ministry of Education, University and Research (MIUR) through the "Dipartimenti di eccellenza" project Science of the Universe.

\section{References}

Ahumada, J., \& Lapasset, E. 1995, A\&AS, 109, 375

Ahumada, J. A., \& Lapasset, E. 2007, A\&A, 463, 789

Albrow, M. D., Gilliland, R. L., Brown, T. M., et al. 2001, ApJ, 559, 1060

Andronov, N., Pinsonneault, M. H., \& Terndrup, D. M. 2006, ApJ, 646, 1160

Arenou, F., Luri, X., Babusiaux, C., et al. 2018, A\&A, 616, A17

Auriere, M., Lauzeral, C., \& Ortolani, S. 1990, Nature, 344, 638

Banks, T., Yontan, T., Bilir, S., \& Canbay, R. 2020, JApA, 41, 6

Bhattacharya, S., Vaidya, K., Chen, W. P., \& Beccari, G. 2019, A\&A, 624, A26

Boffin, H. M. J., Carraro, G., \& Beccari, G. 2015, Ecology of Blue Straggler Stars, 413

Bossini, D., Vallenari, A., Bragaglia, A., et al. 2019, A\&A, 623, A108

Breger, M., \& Wheeler, J. C. 1980, PASP, 92, 514

Bressan, A., Marigo, P., Girardi, L., et al. 2012, MNRAS, 427, 127

Cantat-Gaudin, T., \& Anders, F. 2020, A\&A, 633, A99

Cantat-Gaudin, T., Jordi, C., Vallenari, A., et al. 2018, A\&A, 618, A93

Carraro, G., Vázquez, R. A., \& Moitinho, A. 2008, A\&A, 482, 777

Carraro, G., de Silva, G., Monaco, L., Milone, A. P., \& Mateluna, R. 2014, A\&A, 566, A39

Carrera, R., Bragaglia, A., Cantat-Gaudin, T., et al. 2019, A\&A, 623, A80

Chen, X., \& Han, Z. 2008, MNRAS, 384, 1263

Chen, X., \& Han, Z. 2009, MNRAS, 395, 1822

Clark, L. L., Sandquist, E. L., \& Bolte, M. 2004, AJ, 128, 3019

da Silveira, M. D., Pereira, C. B., \& Drake, N. A. 2018, MNRAS, 476, 4907

Davies, M. B., Benz, W., \& Hills, J. G. 1994, ApJ, 424, 870

Dias, W. S., Alessi, B. S., Moitinho, A., \& Lépine, J. R. D. 2002, A\&A, 389, 871

Eggen, O. J. 1983, AJ, 88, 386

Eggen, O. J., \& Iben, I. 1988, in Progress and Opportunities in Southern Hemisphere Optical Astronomy. The CTIO 25th Anniversary Symposium, eds. V. M. Blanco, \& M. M. Phillips, ASP Conf. Ser., 1, 239

Evans, D. W., Riello, M., De Angeli, F., et al. 2018, A\&A, 616, A4

Ferraro, F. R., Pecci, F. F., Cacciari, C., et al. 1993, AJ, 106, 2324

Ferraro, F. R., Lanzoni, B., Dalessandro, E., et al. 2012, Nature, 492, 393

Ford, E. B., Kozinsky, B., \& Rasio, F. A. 2000, ApJ, 535, 385

Gaia Collaboration (Brown, A. G. A., et al.) 2018, A\&A, 616, A1

Geller, A. M., Mathieu, R. D., Harris, H. C., \& McClure, R. D. 2008, AJ, 135, 2264

Gosnell, N. M., Mathieu, R. D., Geller, A. M., et al. 2014, ApJ, 783, L8
Gosnell, N. M., Mathieu, R. D., Geller, A. M., et al. 2015, ApJ, 814, 163

Hills, J. G., \& Day, C. A. 1976, Astrophys. Lett., 17, 87

Katz, D., Sartoretti, P., Cropper, M., et al. 2019, A\&A, 622, A205

Landsman, W., \& Simon, T. 1998, Am. Astron. Soc. Meeting Abstr., 193, 37.03

Landsman, W., \& Stecher, T. P. 1997, AIP Conf. Ser., 408, 390

Lanzoni, B., Dalessandro, E., Perina, S., et al. 2007, ApJ, 670, 1065

Lapasset, E., \& Ahumada, J. 1996, A\&A, 314, 448

Leiner, E. M., \& Geller, A. 2021, ApJ, 908, 229

Leiner, E., Mathieu, R. D., Stello, D., Vanderburg, A., \& Sandquist, E. 2016, ApJ, 832, L13

Lindegren, L., Hernández, J., Bombrun, A., et al. 2018, A\&A, 616, A2

Mapelli, M., Sigurdsson, S., Ferraro, F. R., et al. 2006, MNRAS, 373, 361

Martinez, C. F., Holanda, N., Pereira, C. B., \& Drake, N. A. 2020, MNRAS, 494 1470

Mathieu, R. D., \& Geller, A. M. 2015, Ecology of Blue Straggler Stars, 413, 29

Mathieu, R. D., \& Latham, D. W. 1986, AJ, 92, 1364

Mathieu, R. D., Latham, D. W., \& Griffin, R. F. 1990, AJ, 100, 1859

McCrea, W. H. 1964, MNRAS, 128, 147

Mermilliod, J. C., Andersen, J., Latham, D. W., \& Mayor, M. 2007, A\&A, 473, 829

Milone, A. A. E., \& Latham, D. W. 1994, AJ, 108, 1828

Milone, A. P., Piotto, G., Bedin, L. R., et al. 2012, A\&A, 540, A16

Momany, Y., Held, E. V., Saviane, I., et al. 2007, A\&A, 468, 973

Monteiro, H., \& Dias, W. S. 2019, MNRAS, 487, 2385

Naoz, S., \& Fabrycky, D. C. 2014, ApJ, 793, 137

Nazé, Y., \& Rauw, G. 2008, A\&A, 490, 801

Perets, H. B., \& Fabrycky, D. C. 2009, ApJ, 697, 1048

Piotto, G., De Angeli, F., King, I. R., et al. 2004, ApJ, 604, L109

Platais, I., Melo, C., Fulbright, J. P., et al. 2008, MNRAS, 391, 1482

Preston, G. W., \& Sneden, C. 2000, AJ, 120, 1014

Rain, M. J., Carraro, G., Ahumada, J. A., et al. 2020, AJ, 159, 59

Rain, M. J., Carraro, G., Ahumada, J., et al. 2021, AJ, 161, 37

Sabbi, E., Ferraro, F. R., Sills, A., \& Rood, R. T. 2004, ApJ, 617, 1296

Sales Silva, J. V., Peña Suárez, V. J., Katime Santrich, O. J., et al. 2014, AJ, 148, 83

Salinas, R., Jílková, L., Carraro, G., Catelan, M., \& Amigo, P. 2012, MNRAS, 421,960

Sandage, A. R. 1953, AJ, 58, 61

Sandquist, E. L., Latham, D. W., Shetrone, M. D., \& Milone, A. A. E. 2003, AJ, 125,810

Santucci, R. M., Placco, V. M., Rossi, S., et al. 2015, ApJ, 801, 116

Stryker, L. L. 1993, PASP, 105, 1081

Tian, B., Deng, L., Han, Z., \& Zhang, X. B. 2006, A\&A, 455, 247

Vaidya, K., Rao, K. K., Agarwal, M., \& Bhattacharya, S. 2020, MNRAS, 496, 2402

Verbunt, F., \& Hut, P. 1987, in The Origin and Evolution of Neutron Stars, eds. D. J. Helfand, \& J. H. Huang, 125, 187

Walborn, N. R. 1979, PASP, 91, 442

Yontan, T., Bilir, S., Bostanc1, Z. F., et al. 2019, Ap\&SS, 364, 152

Zaggia, S. R., Piotto, G., \& Capaccioli, M. 1997, A\&A, 327, 1004 